\title{
siRNA, miRNA and HIV: promises and challenges
}

\author{
Man Lung YEUNG ${ }^{1}$, Yamina BENNASSER ${ }^{1}$, Shu Yun $\mathrm{LE}^{2}$, Kuan Teh JEANG ${ }^{1, *}$ \\ ${ }^{1}$ Molecular Virology Section, Laboratory of Molecular Microbiology, National Institute of Allergy and Infectious Diseases, \\ National Institutes of Health Bethesda, Maryland 20892-0460, USA \\ ${ }^{2}$ Center for Cancer Research Nanobiology Program NCI Center for Cancer Research, NCI, National Institutes of Health, \\ Fredrick, MD 21702, USA
}

\begin{abstract}
Small interfering RNA (siRNA) and microRNA (miRNA) are small RNAs of 18-25 nucleotides (nt) in length that play important roles in regulating gene expression. They are incorporated into an RNA-induced silencing complex (RISC) and serve as guides for silencing their corresponding target mRNAs based on complementary base-pairing. The promise of gene silencing has led many researchers to consider siRNA as an anti-viral tool. However, in long-term settings, many viruses appear to escape from this therapeutical strategy. An example of this may be seen in the case of human immunodeficiency virus type-1 (HIV-1) which is able to evade RNA silencing by either mutating the siRNAtargeted sequence or by encoding for a partial suppressor of RNAi (RNA interference). On the other hand, because miRNA targeting does not require absolute complementarity of base-pairing, mutational escape by viruses from miRNAspecified silencing may be more difficult to achieve. In this review, we discuss stratagems used by various viruses to avoid the cells' antiviral si/mi-RNA defenses and notions of how viruses might control and regulate host cell genes by encoding viral miRNAs (vmiRNAs).
\end{abstract}

Keywords: small interfering RNA, microRNA, RNA interference, human immunodeficiency virus type-1, RNA-induced silencing complex, suppressor of siRNA, viral miRNA.

\section{INTRODUCTION}

The recent discovery of small interfering RNA (siRNA) revealed an important role for small RNAs in regulating gene expression. First described in plants, as "posttrancriptional gene silencing" (PTGS) [1], RNA interference (RNAi) is a nucleic-acid based immune defense against viruses, transgenes and transposons [2]. Triggered by double-stranded RNA (dsRNA), RNAi leads to the sequence specific degradation of a target mRNA [3]. In eukaryotic cells, long dsRNAs are processed by Dicer, an RNAse III like protein, into small RNA duplexes of 21 to 23 nucleotides, called siRNA [4, 5] (Fig. 1). A cellular protein called TAR RNA-binding protein (TRBP) then interacts with and shuttles the Dicer-siRNA complex into the RNA-induced silencing complex (RISC) which con-

\footnotetext{
*Correspondence: Kuan Teh JEANG

Building 4, Room 306, 9000 Rockville Pike, Bethesda, MD 20892 0460, USA.

Tel: 301-496-6680; Fax: 301-480-3686;

E-mail:kj7e@nih.gov
}

tains another protein called Argonaute 2 (Ago2) [6, 7]. While other components of RISC remain to be identified, the current view is that one processed strand of the siRNA is incorporated into the protein complex and serves as a guide sequence for recognition of target RNAs. Thus, an active RISC consists minimally of the Ago 2 protein and the single-stranded siRNA guide [8-11]. When a perfect base-pairing of the single-stranded guide RNA with the target mRNA is made, the PIWI domain of the Ago 2 protein is brought into proximity and cleaves the target mRNA resulting in a post-transcriptional gene silencing [12, 13].

In addition to post-transcriptional RNA silencing, components of the RNA interference pathway are also postulated to be involved in transcriptional gene silencing through RNA-dependent DNA methylation [14]. siRNAs which originate from bidirectional transcription of a cell's target loci are incorporated into an RNA-induced initiator of transcriptional gene silencing (RITS) complex. The composition of RITS is variable from one species to another, and its components are not completely identified. In plants and fission yeast, RITS includes Dicer, Agol (an Argonaute 


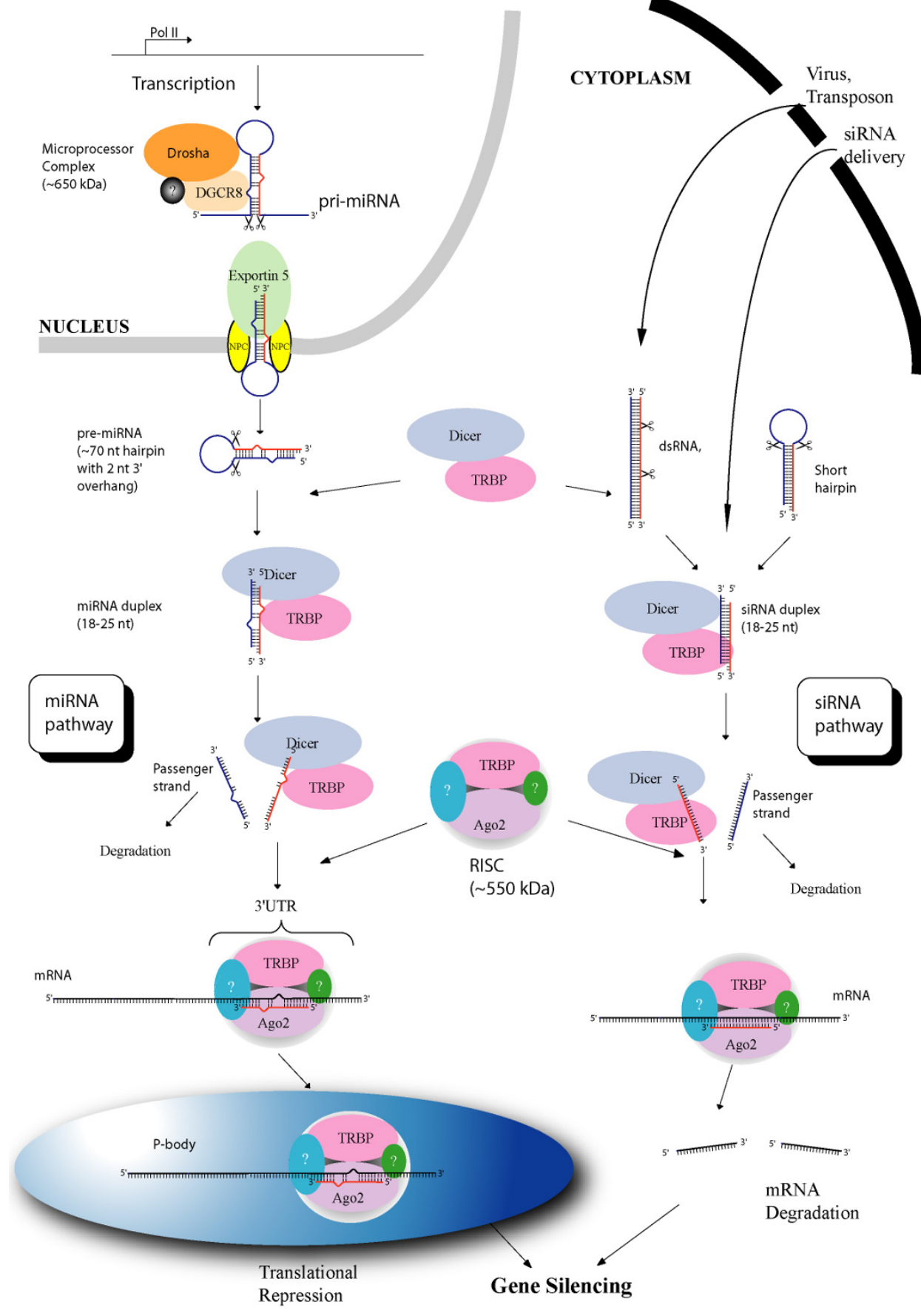

Fig. 1 A comparison of the miRNA and siRNA pathways. miRNAs are derived from large highly-structured precursors pri-miRNAs transcribed by RNA Pol II in the nucleus. After cropping by the Microprocessor, a complex containing Drosha and DGCR8, the primiRNAs are processed into $\sim 70 \mathrm{nt}$ hairpin with $2 \mathrm{nt}$ at the 3' overhang (pre-miRNAs). With the aid of Exportin 5, the pre-miRNAs are then exported into the cytoplasm. In the cytoplasm, both pre-miRNAs and viral/transposon-derived dsRNAs/short hairpins share a common processing pathway which mainly involves Dicer/TRBP and RISC. The loop regions of pre-miRNA and the dsRNAs/short hairpins are trimmed by Dicer to generate miRNA and siRNA duplexes, respectively. One strand of the duplexes is incorporated into the RISC and serves as a guide for RNA silencing. The remaining RNA strand (passenger strand) is then targeted for degradation. Although both miRNAs and siRNAs utilize RISC for gene silencing, mRNAs with perfect complementary to the siRNAs are targeted to degradation while transcripts captured by miRNA-incorporated Ago2 are directed into the P-body resulting in translational repression. NPC: Nuclear Pore Complex 
homolog), Chp1 a chromodomain protein and an RNAdependent RNA polymerase (RdRP) which so far has no known equivalent analogue in vertebrates or insects [15]. It remains unclear whether RITS binds chromatin through a targeted nascent transcript, or through DNA, and then it is envisioned that a protein complex including a methyltransferase that methylates histone 3 methyl 9 (H3K9) is recruited to the promoter. H3K9 methylation consolidates RITS/chromatin complex and initiates the methylation of $\mathrm{CpG}$ islands resulting in transcriptional repression. Although apparently well-described in lower eukaryotes; currently, it remains uncertain whether RITS is fully operative in higher eukaryotes.

Similar to siRNA, another kind of small RNA, microRNA (miRNA) of 18-25 nt, has also been identified to interfere with gene expression [16-18]. miRNAs were first described in Caenorhabditis elegans (C. elegans) to regulate developmental timing [19]. To date, the miRNA Registry database (http://www.sanger.ac.uk/Software/ Rfam/mirna) records 2909 miRNA sequences including 78 in Drosophila melanogaster, 114 in C. elegans, 321 in Homo sapiens [20]. A miRNA is derived from the transcription of a large highly-structured precursor (primiRNA) of $\sim 70 \mathrm{nt}$ encoded by a cellular gene. The primiRNA is then processed in the cell's nucleus into an imperfect shorter stem-loop structure (pre-miRNA) by the Microprocessor, a large complex that includes Drosha and the RNA binding protein DGCR8 [21]; pre-miRNAs are then exported into the cytoplasm by Exportin 5 (Fig. 1). In the cytoplasm, the loop region of the pre-miRNA is cleaved by Dicer to generate an imperfect dsRNA of 18$25 \mathrm{nt}$ in length. Like siRNA, one strand of the doublestranded miRNA is incorporated into the RISC which then becomes armed to silence gene expression [22]. In contrast to siRNA, perfect complementarity is not necessary for miRNA to target its transcripts. One miRNA is, therefore, able to target a number of different transcripts. Thus, a microarray analysis has recently shown that the cellular miRNA, miR-124, can regulate up to $\sim 100$ mRNAs [23]. Conversely, various miRNAs can silence a gene by targeting different portions of the same mRNA. Although both siRNA and miRNA utilize the same machinery (RISC) for gene silencing, the fates of their target mRNAs are not the same. siRNAs shut down gene expression at a post-transcriptional level through mRNA degradation, while miRNAs silence their target genes mainly through translational repression. Once the target transcript is bound to the miRNA-armed Ago2 (within RISC), the entire complex is directed into a ribosome-free compartment named "P-body" [24, 25]. Sequestration of the transcript into the P-body results in a blockage of translation (Fig. 1).

\section{RNA INTEFERENCE: HIV-1 AS AN EXAMPLE}

Recently, RNA silencing has become widely adopted as an experimental tool to inhibit the expression of cellular and viral genes. Accordingly, several siRNAs have been designed to target HIV infection [26]. The first HIV targets were essential genes: such as the gag structural protein [27], the infectivity factors vif and nef [28], and the post-transcriptional regulatory protein, rev [29]. Using siRNA, many investigators reported sequence-specific silencing of targets and observed a robust inhibition of HIV replication during single round infection or short term tissue culturing of virus spanning 3 to 4 days. In addition to targeting HIV genes (nef, tat, gag, vif, env) [26, 30, 31], some have also targeted cellular proteins important to the HIV-1 life cycle. Thus, siRNAs were designed and tested against different steps of virus replication: i) at the entry level to HIV coreceptors CCR5 and CXCR4 [32-34]; ii) at the pre-integration step to the Arp2/3 complex responsible for actin polymerization involved in the routing of HIV toward the nucleus [35], and to PARP-1 [poly (ADPribose) polymerase-1] an abundant nuclear enzyme required for HIV integration [36]; iii) to cyclin T1, CDK9 and SPT5 which function at the level of HIV transcription for elongation of RNA polymerase II on the HIVLTR $[37,38]$, and iv) to other key components such as cyclophilin A [39] and cdk2 [40]. All these targets were found to be efficiently inhibited by transfection of specific siRNAs, and their inhibition correlated with reduction in HIV replication in tissue culture [41]. However, one potentially noteworthy limitation to these findings is the relatively short duration of the assays for efficacy. Indeed, contrary to PTGS in plants which have an autoamplification step, in mammals the effects of siRNAs are transient. One major obstacle remains how to continuously express siRNA in cells and whether sustained inhibition of viruses can be achieved in mammalian cells. A solution for continuous expression is addressed partially by the use of short hairpin sequences (shRNA) which can be processed intracellularly by the cell's Dicer [29, 42]. Whether sustained silencing can be durably maintained by exogenously introduced shRNA-expression vectors remain to be established.

\section{ESCAPE OF HIV-1 FROM RNA INTERFER- ENCE}

We and others have recently reported that HIV can escape from siRNA-mediated gene silencing. Many studies have described that HIV escapes from RNA interference through nucleotide mutations [43]. Das et al. observed that long term targeting of $n e f$ sequence using a lentiviral vector which expressed a suppressive shRNA eventually 
selected for HIV with nucleotide mutations that escaped RNA interference [44]. Sequencing of the emerged RNAiresistant virus showed that the $n e f$ sequence was altered in several ways: in some cases the siRNA recognition site contained several nucleotide substitutions which disrupted base-pairing and in other cases the target was simply deleted. Similar mutation patterns were also observed when Tat was targeted by exogenously introduced shRNA in a stable cell line [45]. Intriguingly, in a more recent report, a different mutational strategy to escape from RNAi was revealed by HIV-1. In this latter study, mutated viruses which showed high level of resistance to RNAi, evolved substitutions, near or in the targeted RNA sequence, which induced an alternative RNA folding resulting in a shielding of the target sequence from recognition by the guide RNA in RISC [46].

How to counteract the viruses' ability to mutate and escape from RNAi is a prime challenge which impedes the development of an efficient and long lasting anti-viral therapy. One idea is to administer several siRNAs targeted to different viral sequences simultaneously. In the case of hepatitis $\mathrm{C}$ virus (HCV) the combined use of two siRNAs limited the emergence of escape mutants seen when each siRNA was used separately [47]. Recently, a computational model was designed to predict viral mutations generated by HIV infection of cells which express RNAi targeted to the virus [48]. Such a tool may be helpful for designing multiple anti-HIV siRNAs which could be used combinatorially [49]. Indeed, the ability of HIV to escape from siRNA reflects another facet of the wellknown mutability of this virus for changing its amino acid sequence to avoid immune clearance in vivo [2]. Unless better strategies are employed, all extant data suggest that singular targeting of individual siRNAs to HIV will always provoke rapid emergence of resistant virus [50-52].

Because HIV-1 appears able to escape facilely from

Tab. 1 Summary of the known virus-encoded suppressors in plant and animal viruses

\begin{tabular}{|c|c|c|}
\hline & Virus & Virus family \\
\hline \multicolumn{3}{|c|}{ Plant viral suppressors of RNA silencing } \\
\hline Beta- C1 & Tomato leaf curl china virus & Begomavirus \\
\hline $\mathrm{CP}$ & Turnip crinkle virus & Carmovirus \\
\hline S protein & Cowpea mosaic virus & Comovirus \\
\hline p21 & Beet yellows virus & Closterovirus \\
\hline $2 b$ & Cucumber mosaic virus & Cucumovirus \\
\hline virus $\mathrm{P} 14$ & Beet necrotic yellow vein & Furovirus \\
\hline $\mathrm{AC} 2$ & African cassava mosaic virus & Geminivirus \\
\hline $\mathrm{C} 2$ & Mungbean yellow mosaic virus & Geminivirus \\
\hline gb & Barley stripe mosaic virus & Hordeivirus \\
\hline P15 & Peanut clump virus & Pecluvirus \\
\hline Pns10 & Rice dwarf phytoreovirus & Phytoreovirus \\
\hline PO & Beet western yellows virus & Polerovirus \\
\hline p25 & Potato virus $\mathrm{X}$ & Potexvirus \\
\hline HC-Pro & Potato virus Y & Potyvirus \\
\hline P1 & Rice yellow mottle virus & Sobemovirus \\
\hline NS3 & Rice hoja blanca virus & Tenuivirus \\
\hline P19 & Tomato bushy stunt virus & Tombusvirus \\
\hline $\mathrm{p} 30$ & Tobacco mosaic virus & Tombamovirus \\
\hline NSs & Tomato spotted wilt virus & Tospovirus \\
\hline p69 & Turnip yellow mosaic virus & Tympvirus \\
\hline \multicolumn{3}{|c|}{ Animal viral suppressors of RNA silencing } \\
\hline VA1 & Adenovirus & Adenovirus \\
\hline Tat & Human Immunodeficiency Virus & Retrovirus \\
\hline Tas & Foamy virus type 1 & Retrovirus \\
\hline B2 & Flock house virus & Nodavirus \\
\hline NS1 & Influenza A & Orthomyxovirus \\
\hline E3L & vaccinia virus & Poxvirus \\
\hline
\end{tabular}


HIV-1
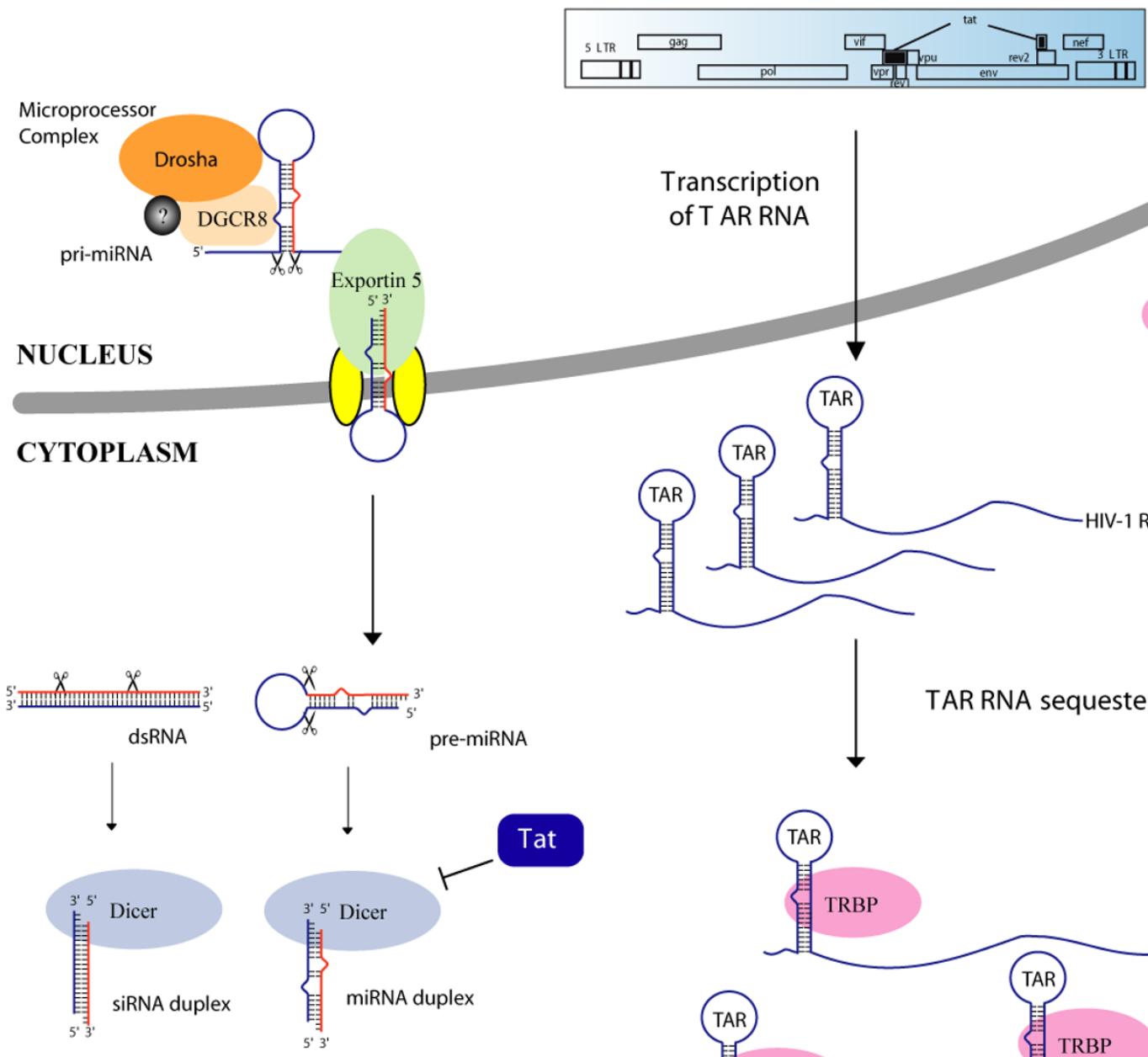

TRBP-less RISC is nonfunctional

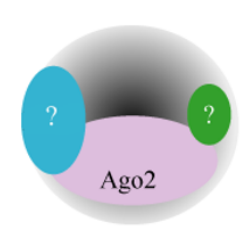

Inactive RISC

(no TRBP)
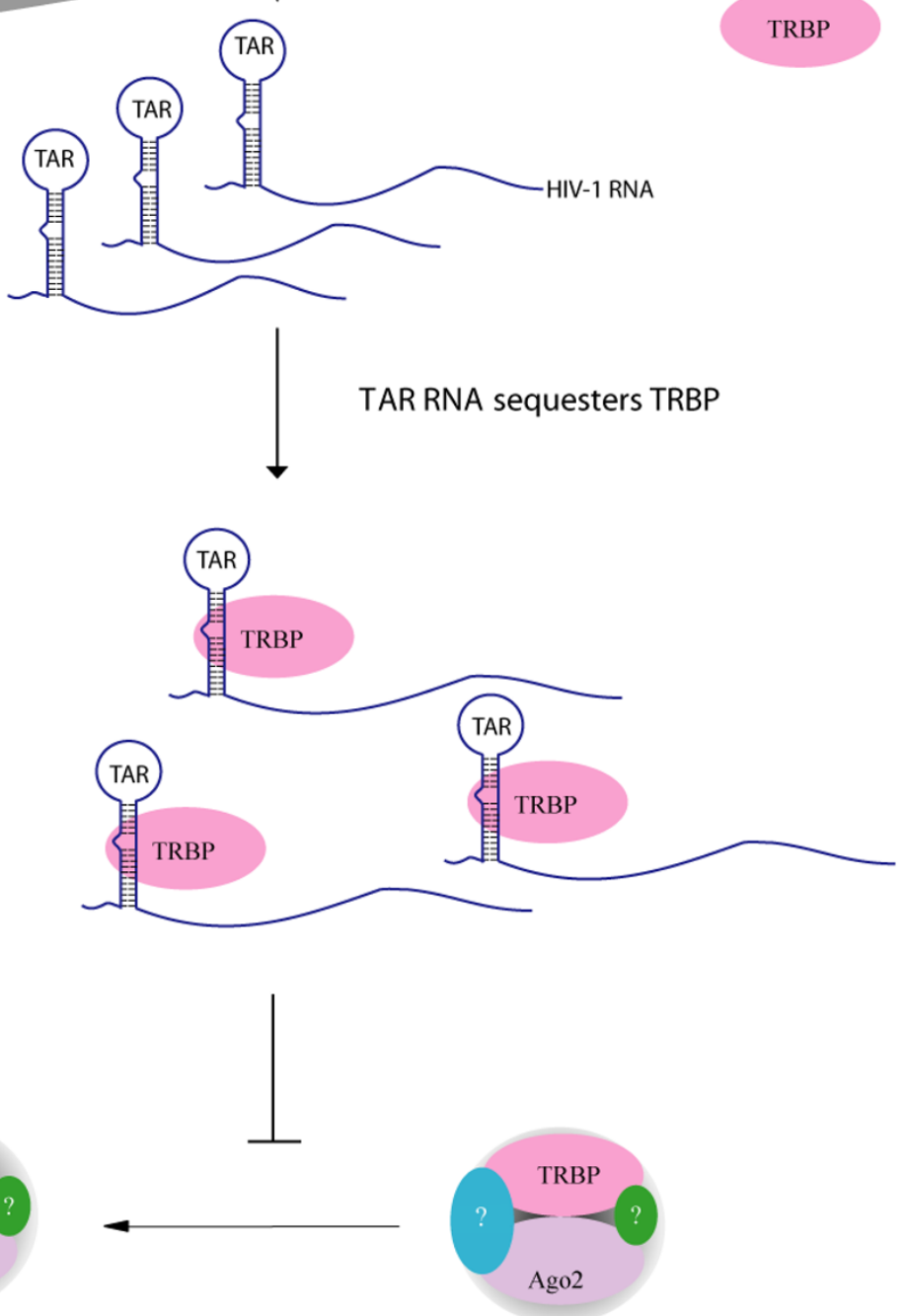

Active RISC

(+ TRBP)

Fig. 2 Interference of the RNA silencing pathway by HIV-TAR RNA. TRBP is a Dicer-interacting protein which leads the Dicerprocessed dsRNA to Ago2 in the RISC. In HIV-1 infected cells, TRBP is envisioned to be sequestered by TAR, a small hairpin leader RNA which is abundantly transcribed by HIV-1 (right). TAR-mediated depletion of TRBP prevents the formation of a functional RISC. On the left, a schematic is shown in which HIV-1 encoded Tat protein is postulated to directly interfere with Dicer function and thus leading to the suppression of the RNA silencing pathway. 
exogenously targeted siRNA, it was highly unexpected that when we used a computer-directed systematic search over the entire HIV-1 genome, we found that the virus maintained a single region of duplexing that could serve as a virus-endogenous shRNA (vshRNA) [50]. Overexpression of this vshRNA was found to inhibit HIV replication. Because this region is conserved among different HIV strains, we hypothesize that there may be unknown selective pressure as to why HIV has not mutated this duplex. If there is indeed a functional constraint that prohibits HIV-1 from altering this vshRNA duplex, then this site could represent an "immutable" sequence to which exogenously introduced siRNA could be targeted efficaciously and durably.

If a virus contains a double-stranded RNA sequence that it cannot mutate, then in order for the virus to escape an induced RNAi, it likely needs to encode an RNAisuppressor. In addition to its ability to generate nucleotide mutations, HIV also appears to utilize a suppressorstrategy to combat the cell's RNAi-defense. This latter discovery came to us serendipitously; our experimental findings suggested that the HIV-encoded Tat protein works to suppress RNA silencing in human cells [50]. Mechanistically, we observed that Tat inhibits Dicer activity in vitro. In the context of intracellular infection, Tat's inhibition of siRNA-processing by Dicer is partially effective as evidenced by the fact that a Tat-deleted virus processed the virus encoded vshRNA more effectively than a Tat-expressing wild type HIV-1 [50]. We note with great interest that a retrovirus related to HIV, human Tcell leukemia virus (HTLV-1) [53], has been described to encode an anti-sense transcript in the $3^{\prime}$ end of its genome [54]. While the exact function of this transcript remains to be fully clarified, we would predict that this HTLV-1 anti-sense RNA would form with its counterpart sense viral transcript a double-stranded siRNA precursor. If our prediction is correct, then HTLV-1, like HIV-1, also maintains a viral siRNA. Moreover, by inference, HTLV1 would need to encode (or co-opt) a yet-identified suppressor of RNAi.

The concept of a virus-encoded suppressor of RNAi is not new. The first RNA silencing suppressors were described in plant viruses $\{$ i.e. the $\mathrm{P} 1 /$ HcPro polypeptide in Tobacco etch potyvirus (TEV) [55] and p19 protein in Tomato bushy stunt virus (TBSV) [56]\}. To date 20 viral suppressors of RNA silencing have been described in plant viruses and 6 in animal viruses $[2,57,58]$ (Tab. 1). These proteins do not conserve any sequence homology with each other, and many are dsRNA binding protein. In addition to direct dsRNA binding which can be understood to inhibit the processing of siRNAs, the various suppressors appear to act at different steps of the RNA interfer- ence pathway. For example, after homodimerization, the p19 tombusvirus protein binds to siRNA and prevents its incorporation into the RISC [59, 60]. By contrast, the Potyviruses HcPro binds siRNA not to influence its association with RISC, but to block its functional activity [61].

One additional way that HIV might interfere with the RNA silencing pathway comes from the identification of TRBP as an essential component of the RNA interference machinery $[6,7,62]$. TRBP was first cloned and identified as a TAR RNA-binding protein [63]. TRBP interacts with Dicer and directs the siRNA-Dicer complex to Ago2 the catalytic component of RISC. Knockdown of TRBP results in destabilization of Dicer and a loss in miRNA biogenesis [6]. Because TAR RNA is a high-affinity ligand for TRBP, one can imagine that when HIV-1 expresses TAR RNA this viral RNA molecule could attach to TRBP and making it unavailable for use by the Dicer-siRNARISC complex. Thus both Tat and TAR may represent virus-encoded moieties that act to suppress Dicer and/or RISC function (Fig. 2).

\section{FUNCTIONAL ROLES FOR KNOWN MIRNA}

Although similar to siRNA, miRNAs are derived from endogenous RNAs that contain stem-loop structures. miRNAs have been described in a variety of species including invertebrates, plants, insects and mammals [64]. To date, 321 human miRNAs are listed in the miRNA Registry database. Some miRNAs function to regulate developmental timing, signal transduction, apoptosis, cell proliferation and tumorigenesis $[65,66]$ (Tab. 2). Interestingly, a cellular miRNA (miR-32) was recently identified to inhibit infection by the primate foamy virus type 1 (PFV-1) [67]. Knock-down of miR-32 expression by anti-sense oligonucleotides or deletion of the miRNA target sequence from the viral genome significantly enhanced PFV-1 replication. This finding provides the first evidence that cells may use miRNA as an anti-viral defense. Of interest, PFV-1 was found to encode a suppressor of miRNA in its Tas protein. Thus, it was found that Tasexpressing cells showed increased PFV-1 production when compared with the Tas-nonexpressing cells [67-69]. PFV1 's infectivity therefore depends on three factors: the tissue-specific miRNA expression pattern (i.e. miR-32), the expression of PFV-1 encoded viral silencing suppressor (i.e. Tas), and the ability of PFV-1 to mutate its viral sequence targeted by miR-32. Above, we pointed out the interesting parallel that HIV encodes a Tat protein which appears to partially suppress Dicer's function [50]. One intriguing unanswered question is whether there are some cellular miRNAs that target HIV, and if so whether HIV1 Tat could also be a miRNA-suppressor.

In a different context, the human miRNA, miR-122 is 
Tab. 2 Functions of known miRNAs

\begin{tabular}{|c|c|c|c|c|}
\hline Biological Function of miRNAs & miRNA & Target gene(s) & Species & Refs \\
\hline \multirow[t]{12}{*}{ Developmental Timing } & lin-4 & lin-14 & $\mathrm{Ce}$ & {$[87,88]$} \\
\hline & & $\operatorname{lin}-28$ & & [89] \\
\hline & let-7 & $\operatorname{lin}-41$ & $\mathrm{Ce}$ & {$[90,91]$} \\
\hline & & hbl-1 & & {$[92,93]$} \\
\hline & 1sy-6 & $\operatorname{cog}-1$ & $\mathrm{Ce}$ & {$[94]$} \\
\hline & miR-172 & AP2 & At & {$[68,95-97]$} \\
\hline & miR-JAW & TCP4 & At & {$[98]$} \\
\hline & miR-159 & MYB33 & At & {$[95,98]$} \\
\hline & $\operatorname{miR}-7$ & HLHm3 & $\mathrm{Dm}$ & {$[99,100]$} \\
\hline & & hairy & & {$[100]$} \\
\hline & & $\mathrm{m} 4$ & & {$[100]$} \\
\hline & miR-273 & die-1 & $\mathrm{Ce}$ & {$[101]$} \\
\hline \multirow[t]{4}{*}{ Apoptosis } & $\operatorname{miR}-14$ & grim & $\mathrm{Dm}$ & {$[100,102]$} \\
\hline & & reaper & & {$[100,102]$} \\
\hline & & sickle & & {$[100,102]$} \\
\hline & bantam miRNA & hid & $\mathrm{Dm}$ & {$[103]$} \\
\hline \multirow[t]{2}{*}{ Cell proliferation } & miR-101 & ENX-1 & $\mathrm{Hs}$ & [104] \\
\hline & & N-MYC & & [104] \\
\hline Tumorigenesis & miR-19a & PTEN & & [104] \\
\hline Signal transduction & miR-375 & Myotrophin & $\mathrm{Mm}$ & [105] \\
\hline
\end{tabular}

Ce, Caenorhabditis elegans; At, Arabidopsis thaliana; Dm, Drosophila melanogaster; Hs, Homo sapiens; Mm, Mus musculus.

specifically expressed in liver cells and was found to be required for efficient Hepatitis $\mathrm{C}$ virus (HCV) replication in hepatocytes [70]. In this case, the miRNA is an activator of the viral infection. It is thought that miR-122 acts through binding to the 5' non-coding region of the $\mathrm{HCV}$ RNA, although miR-122's exact mechanism of action remains unknown. The effect of miR-122 appears to be tissue specific and not absolutely essential since in cells that do not express miR-122 (i.e. non-hepatic cells), HCV replication can be documented. It remains to be seen if miR-122 is a unique example or whether other cellular miRNAs could target 5' non-coding region of viruses and contribute to facilitated replication of pathogens.

\section{IDENTIFICATION OF VIRAL MIRNA}

Recent reports suggest that viruses can also encode miRNAs. To date, a total of 48 viral miRNAs (vmiRNAs) have been described (Tab. 3) in herpesviruses [71-73], polymoaviruses (SV40) [74] and retroviruses (HIV-1) [75, 76]. Most of the vmiRNAs were identified by small RNA cloning [71-73, 75]. A rare subset of vmiRNAs were predicted by computational algorithm and then verified by Northern blotting. In HIV-1, 5 stem-loop structures that can potentially be processed into miRNAs were predicted computationally in one report [77]. In a second report, a vmiRNA encoded by the nef region of HIV-1 termed miRN367 was physically identified and isolated [75].

Size-fractionated small RNAs can be isolated and cloned from cells. Methodologically, RNAs of around 18-25 nt can be first purified by excision from acrylamide gel. To generate cDNAs from the purified small RNAs, individual linkers are ligated respectively to the $3^{\prime}$ and 5 ' ends. Using the linkers, the small RNAs are reverse transcribed and then PCR amplified. Subsequently, restriction digestion of the linker sequences allows for the cloning of the small RNAs into plasmids. Sequencing of the cloned plasmids identifies small RNAs including miRNAs in cells.

A second way to identify miRNA is through computerdriven predications. Computational prediction uses an algorithm that recognizes the stem-loop structures present in the pri- and pre-miRNA. The program can scan large amounts of sequences and identify candidate stretches that can form stable local stem-loop structures. Based on the sequence and structural features conserved in known miRNAs, predicted miRNA-candidates are then selected and can be physically validated by Northern blotting. We 
Tab. 3 Summary of the identified small RNAs encoded by viruses

\begin{tabular}{|c|c|c|c|}
\hline Species & No. of Stem-loop & stem-loop & miRNA sequence \\
\hline \multirow[t]{6}{*}{ Epstein Barr virus (EBV) } & 5 & ebv-miR-BART1 & ucuuaguggaagugacgugcu \\
\hline & & ebv-miR-BHRF1-1 & uaaccugaucagccccggaguu \\
\hline & & ebv-miR-BHRF1-2 & uaucuuuugcggcagaaauugaa \\
\hline & & ebv-miR-BHRF1-2* & aaauucuguugcagcagauagc \\
\hline & & ebv-miR-BHRF1-3 & uaacgggaaguguguaagcacac \\
\hline & & ebv-miR-BART2 & uauuuucugcauucgcccuugc \\
\hline \multirow[t]{13}{*}{ Human cytomegalovirus } & 11 & hcmv-miR-US5-1 & ugacaagccugacgagagcgu \\
\hline & & hcmv-miR-US5-2 & uuaugauaggugugacgauguc \\
\hline & & hemv-miR-UL22A-1 & uaacuagccuucccgugaga \\
\hline & & hcmv-miR-UL22A-1* & ucaccagaaugcuaguuuguag \\
\hline & & hcmv-miR-US25-1 & aaccgcucaguggcucggacc \\
\hline & & hcmv-miR-US25-2-5p & auccacuuggagagcuccegcgg \\
\hline & & hcmv-miR-US25-2-3p & agcggucuguucagguggauga \\
\hline & & hcmv-miR-US33-1 & gauugugcccggaccgugggcg \\
\hline & & hemv-miR-UL36-1 & ucguugaagacaccuggaaaga \\
\hline & & hemv-miR-UL112-1 & aagugacggugagauccaggcu \\
\hline & & hcmv-miR-UL148D-1 & ucguccuccccuucuucaccg \\
\hline & & hemv-miR-UL70-1 & ugcgucucggccucguccaga \\
\hline & & hcmv-miR-US4-1 & cgacauggacgugcaggggga \\
\hline \multirow{16}{*}{ Kaposi sarcoma-associated herpesvirus } & 12 & kshv-miR-K12-2 & aacuguaguccgggucgaucug \\
\hline & & kshv-miR-K12-1 & auuacaggaaacuggguguaagc \\
\hline & & kshv-miR-K12-10a & uaguguuguccccccgaguggc \\
\hline & & kshv-miR-K12-10b & ugguguuguccccccgaguggc \\
\hline & & kshv-miR-K12-11 & uuaaugcuuagccuguguccga \\
\hline & & kshv-miR-K12-3 & ucacauucugaggacggcagcg \\
\hline & & kshv-miR-K12-3* & ucgcggucacagaaugugaca \\
\hline & & kshv-miR-K12-4-5p & agcuaaaccgcaguacucuagg \\
\hline & & kshv-miR-K12-4-3p & uagaauacugaggccuagcuga \\
\hline & & kshv-miR-K12-5 & uaggaugccuggaacuugccgg \\
\hline & & kshv-miR-K12-6-5p & ccagcagcaccuaauccaucgg \\
\hline & & kshv-miR-K12-6-3p & ugaugguuuucgggcuguugag \\
\hline & & kshv-miR-K12-7 & ugaucccauguugcuggcgcu \\
\hline & & kshv-miR-K12-8 & uaggcgcgacugagagagcacg \\
\hline & & kshv-miR-K12-9 & cuggguauacgcagcugcguaa \\
\hline & & kshv-miR-K12-9* & acccagcugcguaaaccccgcu \\
\hline \multirow[t]{10}{*}{ Mouse gammaherpesvirus 68} & 9 & mghv-miR-M1-1 & uagaaauggccguacuuccuuu \\
\hline & & mghv-miR-M1-2 & cagacccccucucccccucuuu \\
\hline & & mghv-miR-M1-3 & gaggugagcaggaguugcgcuu \\
\hline & & mghv-miR-M1-4 & ucgaggagcacguguuauucua \\
\hline & & mghv-miR-M1-5 & agaguugagaucgggucgucuc \\
\hline & & mghv-miR-M1-6 & ugaaacugugugaggugguuuu \\
\hline & & mghv-miR-M1-7-5p & aaagguggaggugcgguaaccu \\
\hline & & mghv-miR-M1-7-3p & gauaucgegeccaccuuuauu \\
\hline & & mghv-miR-M1-8 & agcacucacuggggguuugguc \\
\hline & & mghv-miR-M1-9 & ucacauuugccuggaccuuuuu \\
\hline Human immunodeficiency virus Type 1 & 1 & $\operatorname{miR}-\mathrm{N} 367$ & acugaccuuuggauggugcuucaa \\
\hline Simian Virus 40 & 2 & SVmiRNA & $\begin{array}{l}\text { ugaggggccugaaaugagccuu } \\
\text { gccuguuucaugcccugagu }\end{array}$ \\
\hline
\end{tabular}


used a computational approach slightly different from the standard miRanda software (http://www.microrna.org/ miranda_new.html) [78] to predict the existence of miRNA candidates in the HIV-1 genome [77]. Once a vmiRNA is predicted, a second level of analysis can be made by scanning a database of human 3' untranslated mRNA regions for sites with complementarity to the vmiRNA. Such an analysis when applied to the 5 predicted vmiRNAs encoded by HIV-1 suggested the existence of $\sim 500$ to 1000 cellular transcripts that can be targeted by the virus [77]. If this analysis can be experimentally validated, then use of virally encoded miRNAs by viruses could be one way that these pathogens alter the landscape of host cell gene expression for their selfish benefit [79].

Despite the above hypothesis, the precise functions of many vmiRNAs are currently unknown. However, predictions of vmiRNA functions based on their potential target genes and their region of transcription have been made. One view is that vmiRNAs are used by viruses to self-moderate viral expression in infected cells; this moderation could assist such cells to escape immune surveillance $[71,74-76]$. This idea is based on the finding that some of the vmiRNA sequences, including HIV-1 miRN367 and EBV miR-BART2, target the coding regions of viral genes $[71,75]$. By targeting their own transcripts, the viruses may attenuate their own pathogenicity and allow for maintenance of chronicity in host organisms. Indeed, ectopic expression of the miR-N367, a HIV-1 $n e f$-derived miRNA, effectively silenced nef expression, and attenuated HIV-1's transcription and replication [75]. Similarly, miR-BART2, a miRNA derived from EpsteinBarr Virus (EBV), targets EBV's DNA polymerase (BALF5) for silencing [71]. Additionally, SV40-encoded miRNAs (SVmiRNAs) are expressed late in infection, and these SVmiRNAs have been found to regulate early viral mRNAs [74]. Interestingly, mutant SV40 deficient in SVmiRNAs accumulated excessive amounts of $T$ antigen and became more susceptible to cytotoxic $\mathrm{T}$ lymphocyte (CTL) response as well as stimulated local cytokine release. Finally, more work is needed to understand how vmiRNAs can regulate cellular gene expression.

\section{DIFFICULTIES IN ISOLATING HIV VIRAL MIRNA}

Currently, the two common methods to identify miRNA are small RNA cloning and computational prediction followed by Northern blot analysis. The ability of these methods to detect miRNAs depends on the abundance of miRNAs and the accuracy of the software for predicting stem-loop secondary structures. Both criteria are challenging for vmiRNA identification because the timing and pattern of vmiRNA expression are unpredictable [79, 80]. Indeed, reports have suggested that some vmiRNAs are only seen during lytic viral replication (EBV, KSHV) [71, 82]. Thus, the ability to capture vmiRNA by small RNA cloning depends on the specific stage of infection, the robustness of infection, and the infected cell type.

Although good success in predicting eukaryotes miRNAs using computational programs has been reported $[71,72,83,84]$, sequence analysis of extant vmiRNAs show that each vmiRNA sequence is often unique and share no sequence homology with other vmiRNAs or with host cell miRNAs. Absent a collection of reliable rules, the accurate computational prediction of vmiRNAs is difficult because the current softwares for predicting cellular miRNAs do rely heavily on cross-species sequence conservation between cells $[82,84,85]$. An illustration of the difficulty with in silico prediction of viral miNRA is shown by the SVM methodology designed by Pfeffer et al. which predicted several herpesvirus vmiRNA [82], but was unable to provide useful vmiRNA predictions for small RNA viruses, including HIV-1. By contrast, 5 HIV-1 vmiRNA candidates were predicted by a different computational algorithm called StemED [77]. In addition to the predicted HIV-1 vmiRNAs, the physical isolation of an HIV-1 vmiRNA, miR-N367, by Omoto et al. [75] suggests that further understanding of the processes for vmiRNA biogenesis and the development of more sensitive and accurate tools for vmiRNA detection, prediction, and isolation are needed.

Finally, much attention has been focused on Drosha because of its role in miRNA maturation. Interestingly, Drosha also plays an important role in pre-ribosomal RNA processing [86]. We have made a curious observation that when we isolate small RNAs from cells, many isolates are fragments of rRNAs (unpublished observation). We note that others have also seen a high proportion of rRNA being isolated during their small RNA cloning attempts [71]. Whether Drosha authentically processes ribosomal RNAs into physiologically relevant small RNAs remains to be established. If the above thinking is found to be correct, then a provocative concept would be that rRNAs may also be precursors for functional small miRNA-like moieties. Perhaps, in addition to siRNAs and miRNAs, there is a third class of biologically active srRNAs, small ribosomal RNAs. Time will tell.

\section{ACKNOWLEDGEMENTS}

This work was supported through intramural funds from the NIAID, and the NCI, NIH.

\section{REFERENCES}

1 Fire A, Xu S, Montgomery MK, et al. Potent and specific ge- 
netic interference by double-stranded RNA in Caenorhabditis elegans. Nature 1998; 391:806-11.

2 Voinnet $O$. Induction and suppression of RNA silencing: insights from viral infections. Nat Rev Genet 2005; 6:206-20.

3 Hannon GJ. RNA interference. Nature 2002; 418:244-51.

4 Zamore PD. Plant RNAi: How a viral silencing suppressor inactivates siRNA. Curr Biol 2004; 14:R198-200.

5 Elbashir SM, Harborth J, Lendeckel W, et al. Duplexes of 21nucleotide RNAs mediate RNA interference in cultured mammalian cells. Nature 2001, 411:494-8.

6 Chendrimada TP, Gregory RI, Kumaraswamy E, et al. TRBP recruits the Dicer complex to Ago 2 for microRNA processing and gene silencing. Nature 2005; 436:740-4.

7 Haase AD, Jaskiewicz L, Zhang H, et al. TRBP, a regulator of cellular PKR and HIV-1 virus expression, interacts with Dicer and functions in RNA silencing. EMBO Rep 2005; 6:961-7.

8 Martinez J, Patkaniowska A, Urlaub H, Luhrmann R, Tuschl T. Single-stranded antisense siRNAs guide target RNA cleavage in RNAi. Cell 2002; 110:563-74.

9 Liu J, Carmell MA, Rivas FV, et al. Argonaute2 is the catalytic engine of mammalian RNAi. Science 2004; 305:1437-41.

10 Martinez J, Tuschl T. RISC is a 5' phosphomonoester-producing RNA endonuclease. Genes Dev 2004; 18:975-80.

11 Meister G, Landthaler M, Patkaniowska A, et al. Human Argonaute2 mediates RNA cleavage targeted by miRNAs and siRNAs. Mol Cell 2004; 15:185-97.

12 Rivas FV, Tolia NH, Song JJ, et al. Purified Argonaute2 and an siRNA form recombinant human RISC. Nat Struct Mol Biol 2005; 12:340-9.

13 Carmell MA, Zhang L, Conklin DS, Hannon GJ, Rosenquist TA. Germline transmission of RNAi in mice. Nat Struct Biol 2003; 10:91-2.

14 Wassenegger M. The role of the RNAi machinery in heterochromatin formation. Cell 2005; 122:13-6.

15 Verdel A, Jia S, Gerber S, et al. RNAi-mediated targeting of heterochromatin by the RITS complex. Science $2004 ; \mathbf{3 0 3}$ :6726.

16 He L, Hannon GJ. MicroRNAs: small RNAs with a big role in gene regulation. Nat Rev Genet 2004; 5:522-31.

17 Bartel DP. MicroRNAs: genomics, biogenesis, mechanism, and function. Cell 2004; 116:281-97.

18 Zamore PD, Haley B. Ribo-gnome: the big world of small RNAs. Science 2005; 309:1519-24.

19 Pasquinelli AE, Ruvkun G: Control of developmental timing by micrornas and their targets. Annu Rev Cell Dev Biol 2002; 18: 495-513.

20 Griffiths-Jones S. The microRNA Registry. Nucleic Acids Res 2004; 32:D109-11.

21 Han J, Lee Y, Yeom KH, et al. The Drosha-DGCR8 complex in primary microRNA processing. Genes Dev 2004; 18:3016-27.

22 Tang G: siRNA and miRNA: an insight into RISCs. Trends Biochem Sci 2005; 30:106-14.

23 Lim LP, Lau NC, Garrett-Engele P, et al. Microarray analysis shows that some microRNAs downregulate large numbers of target mRNAs. Nature 2005; 433:769-73.

24 Liu J, Valencia-Sanchez MA, Hannon GJ, Parker R. MicroRNAdependent localization of targeted mRNAs to mammalian Pbodies. Nat Cell Biol 2005; 7:719-23.

25 Sen GL, Blau HM. Argonaute 2/RISC resides in sites of mam- malian mRNA decay known as cytoplasmic bodies. Nat Cell Biol 2005; 7:633-636.

26 Boden D, Pusch O, Ramratnam B. HIV-1-specific RNA interference. Curr Opin Mol Ther 2004; 6:373-80.

27 Novina CD, Murray MF, Dykxhoorn DM, et al. siRNA-directed inhibition of HIV-1 infection. Nat Med 2002; 8:681-6.

28 Jacque JM, Triques K, Stevenson M. Modulation of HIV-1 replication by RNA interference. Nature 2002; 418:435-8.

29 Lee NS, Dohjima T, Bauer G, et al. Expression of small interfering RNAs targeted against HIV-1 rev transcripts in human cells. Nat Biotechnol 2002; 20:500-5.

30 Capodici J, Kariko K, Weissman D. Inhibition of HIV-1 infection by small interfering RNA-mediated RNA interference. $\mathrm{J}$ Immunol 2002; 169:5196-201.

31 Coburn GA, Cullen BR. Potent and specific inhibition of human immunodeficiency virus type 1 replication by RNA interference. J Virol 2002; 76:9225-31.

32 Anderson J, Banerjea A, Planelles V, Akkina R. Potent suppression of HIV type 1 infection by a short hairpin anti-CXCR4 siRNA. AIDS Res Hum Retroviruses 2003; 19:699-706.

33 Zhou N, Fang J, Mukhtar M, Acheampong E, Pomerantz RJ. Inhibition of HIV-1 fusion with small interfering RNAs targeting the chemokine coreceptor CXCR4. Gene Ther 2004; 11: 1703-12.

34 Anderson J, Akkina R. CXCR4 and CCR5 shRNA transgenic $\mathrm{CD} 34+$ cell derived macrophages are functionally normal and resist HIV-1 infection. Retrovirology 2005,; 2:53.

35 Komano J, Miyauchi K, Matsuda Z, Yamamoto N. Inhibiting the Arp2/3 complex limits infection of both intracellular mature vaccinia virus and primate lentiviruses. Mol Biol Cell 2004; 15: 5197-207.

36 Kameoka M, Nukuzuma S, Itaya A, et al. RNA interference directed against Poly(ADP-Ribose) polymerase 1 efficiently suppresses human immunodeficiency virus type 1 replication in human cells. J Virol 2004; 78:8931-4.

37 Chiu YL, Cao H, Jacque JM, Stevenson M, Rana TM. Inhibition of human immunodeficiency virus type 1 replication by RNA interference directed against human transcription elongation factor P-TEFb (CDK9/CyclinT1). J Virol 2004, 78:251729.

38 Ping YH, Chu CY, Cao H, et al. Modulating HIV-1 replication by RNA interference directed against human transcription elongation factor SPT5. Retrovirology 2004; 1:46.

39 Sokolskaja E, Sayah DM, Luban J. Target cell cyclophilin A modulates human immunodeficiency virus type 1 infectivity. J Virol 2004; 78:12800-8.

40 Ammosova T, Berro R, Kashanchi F, Nekhai S. RNA interference directed to CDK2 inhibits HIV-1 transcription. Virology 2005; 341:171-8.

41 Pomerantz RJ. RNA interference meets HIV-1: will silence be golden? Nat Med 2002; 8:659-60.

42 Lee SK, Dykxhoorn DM, Kumar P, et al. Lentiviral delivery of short hairpin RNAs protects CD4 T cells from multiple clades and primary isolates of HIV. Blood 2005; 106:818-26.

43. Michienzi A, Castanotto D, Lee N, et al. RNA-mediated inhibition of HIV in a gene therapy setting. Ann N Y Acad Sci 2003; 1002:63-71.

44 Das AT, Brummelkamp TR, Westerhout EM, et al. Human immunodeficiency virus type 1 escapes from RNA interference- 
mediated inhibition. J Virol 2004; 78:2601-5.

45 Boden D, Pusch O, Lee F, Tucker L, Ramratnam B. Human immunodeficiency virus type 1 escape from RNA interference. J Virol 2003; 77:11531-5.

46 Westerhout EM, Ooms M, Vink M, Das AT, Berkhout B. HIV1 can escape from RNA interference by evolving an alternative structure in its RNA genome. Nucleic Acids Res 2005; 33:796804.

47 Wilson JA, Richardson CD. Hepatitis C virus replicons escape RNA interference induced by a short interfering RNA directed against the NS5b coding region. J Virol 2005; 79:7050-8.

48 Leonard JN, Schaffer DV. Computational design of antiviral RNA interference strategies that resist human immunodeficiency virus escape. J Virol 2005; 79:1645-4.

49 Leonard JN, Schaffer DV. Antiviral RNAi therapy: emerging approaches for hitting a moving target. Gene Ther 2005 .

50 Bennasser Y, Le SY, Benkirane M, Jeang KT: Evidence that HIV-1 encodes an siRNA and a suppressor of RNA silencing.. Immunity 2005, 22:607-19.

51 Ding SW, Li H, Lu R, Li F, Li WX: RNA silencing: a conserved antiviral immunity of plants and animals. Virus Res 2004, 102: 109-115.

$52 \mathrm{Li} \mathrm{HW}$, Ding SW. Antiviral silencing in animals. FEBS Lett 2005; 579:5965-73

53 Azran I, Schavinsky-Khrapunsky Y, Aboud M. Role of Tax protein in human T-cell leukemia virus type-I leukemogenicity. Retrovirology 2004; 1:20.

54 Gaudray G, Gachon F, Basbous J, et al. The complementary strand of the human T-cell leukemia virus type 1 RNA genome encodes a bZIP transcription factor that down-regulates viral transcription. J Virol 2002, 76:12813-22.

55 Kasschau KD, Carrington JC. A counterdefensive strategy of plant viruses: suppression of posttranscriptional gene silencing. Cell 1998; 95:461-470.

56 Voinnet O, Pinto YM, Baulcombe DC. Suppression of gene silencing: a general strategy used by diverse DNA and RNA viruses of plants. Proc Natl Acad Sci U S A 1999; 96:14147-52.

57 Roth BM, Pruss GJ, Vance VB. Plant viral suppressors of RNA silencing. Virus Res 2004; 102:97-108.

$58 \mathrm{Qu} \mathrm{F}$, Morris TJ. Suppressors of RNA silencing encoded by plant viruses and their role in viral infections. FEBS Lett 2005; 579:5958-64

59 Ye K, Malinina L, Patel DJ. Recognition of small interfering RNA by a viral suppressor of RNA silencing. Nature 2003; 426: 874-8.

60 Lakatos L, Szittya G, Silhavy D, Burgyan J. Molecular mechanism of RNA silencing suppression mediated by 19 protein of tombusviruses. Embo J 2004; 23:876-84.

61 Kasschau KD, Xie Z, Allen E, et al. P1/HC-Pro, a viral suppressor of RNA silencing, interferes with Arabidopsis development and miRNA function. Dev Cell 2003; 4:205-217.

62 Saito K, Ishizuka A, Siomi H, Siomi MC. Processing of premicroRNAs by the Dicer-1-Loquacious complex in Drosophila cells. PLoS Biol 2005; 3:e235.

63 Gatignol A, Buckler-White A, Berkhout B, Jeang KT. Characterization of a human TAR RNA-binding protein that activates the HIV-1 LTR. Science 1991; 251:1597-600.

64 Griffiths-Jones S. The microRNA Registry. Nucleic Acids Res 2004, 32 Database issue:D109-111.
65 Croce $\mathrm{CM}$, Calin GA. miRNAs, cancer, and stem cell division. Cell 2005; 122:6-7.

$66 \mathrm{Kim}$ VN. Small RNAs: classification, biogenesis, and function. Mol Cells 2005; 19:1-15.

67 Lecellier CH, Dunoyer P, Arar K, et al. A cellular microRNA mediates antiviral defense in human cells. Science 2005; 308: $557-60$.

68 Kasschau KD, Xie Z, Allen E, et al. P1/HC-Pro, a viral suppressor of RNA silencing, interferes with Arabidopsis development and miRNA unction. Dev Cell 2003; 4:205-217.

69 Dunoyer P, Lecellier CH, Parizotto EA, Himber C, Voinnet O. Probing the microRNA and small interfering RNA pathways with virus-encoded suppressors of RNA silencing. Plant Cell 2004; 16:1235-50.

70 Jopling CL, Yi M, Lancaster AM, Lemon SM, Sarnow P. Modulation of hepatitis $\mathrm{C}$ virus RNA abundance by a liver-specific MicroRNA. Science 2005; 309:1577-81.

71 Pfeffer S, Zavolan M, Grasser FA, et al. Identification of virusencoded microRNAs. Science 2004; 304:734-6.

72 Pfeffer S, Sewer A, Lagos-Quintana M, et al. Identification of microRNAs of the herpesvirus family. Nat Methods 2005, 2: 269-276.

73 Grey F, Antoniewicz A, Allen E, et al. Identification and characterization of human cytomegalovirus-encoded microRNAs. J Virol 2005; 79:12095-9.

74 Sullivan CS, Grundhoff AT, Tevethia S, Pipas JM, Ganem D. SV40-encoded microRNAs regulate viral gene expression and reduce susceptibility to cytotoxic $\mathrm{T}$ cells. Nature $2005 ; \mathbf{4 3 5}$ : 682-6.

75 Omoto S, Ito M, Tsutsumi Y, et al. HIV-1 nef suppression by virally encoded microRNA. Retrovirology 2004; 1:44.

76 Omoto S, Fujii YR. Regulation of human immunodeficiency virus 1 transcription by nef microRNA. J Gen Virol 2005; 86: $751-5$.

77 Bennasser Y, Le SY, Yeung ML, Jeang KT. HIV-1 encoded candidate micro-RNAs and their cellular targets.Retrovirology 2004; 1:43

78 John B, Enright AJ, Aravin A, et al. Human MicroRNA targets. PLoS Biol 2004; 2 :e363

79 Arendt CW, Littman DR. HIV: master of the host cell. Genome Biol 2001, 2:REVIEWS1030.

80 Lee Y, Kim M, Han J, et al. MicroRNA genes are transcribed by RNA polymerase II. Embo J 2004; 23:4051-60.

81 Cai X, Hagedorn CH, Cullen BR. Human microRNAs are processed from capped, polyadenylated transcripts that can also function as mRNAs. RNA 2004; 10:1957-66.

82 Pfeffer S, Sewer A, Lagos-Quintana M, et al. Identification of microRNAs of the herpesvirus family. Nat Methods 2005; 2: 269-76.

83 Lim LP, Lau NC, Weinstein EG, et al. The microRNAs of Caenorhabditis elegans. Genes Dev 2003; 17:991-1008.

84 Lai EC, Tomancak P, Williams RW, Rubin GM. Computational identification of Drosophila microRNA genes. Genome Biol 2003; 4:R42.

85 Lim LP, Glasner ME, Yekta S, Burge CB, Bartel DP. Vertebrate microRNA genes. Science 2003; 299:1540.

$86 \mathrm{Wu} \mathrm{H}, \mathrm{Xu} \mathrm{H}$, Miraglia LJ, Crooke ST. Human RNase III is a $160-\mathrm{kDa}$ protein involved in preribosomal RNA processing. $\mathrm{J}$ Biol Chem 2000; 275:36957-65. 
87 Lee RC, Feinbaum RL, Ambros V. The C. elegans heterochronic gene lin-4 encodes small RNAs with antisense complementarity to lin-14. Cell 1993; 75:843-54.

88 Wightman B, Ha I, Ruvkun G: Posttranscriptional regulation of the heterochronic gene lin-14 by lin-4 mediates temporal pattern formation in C. elegans. Cell 1993; 75:855-62.

89 Moss EG, Lee RC, Ambros V. The cold shock domain protein LIN-28 controls developmental timing in C. elegans and is regulated by the lin-4 RNA. Cell 1997; 88:637-46.

90 Reinhart BJ, Slack FJ, Basson M, et al. The 21-nucleotide let-7 RNA regulates developmental timing in Caenorhabditis elegans. Nature 2000; 403:901-6.

91 Slack FJ, Basson M, Liu Z, et al. The lin-41 RBCC gene acts in the $\mathrm{C}$. elegans heterochronic pathway between the let-7 regulatory RNA and the LIN-29 transcription factor. Mol Cell 2000; 5:659-69.

92 Lin SY, Johnson SM, Abraham M, et al. The C elegans hunchback homolog, hbl-1, controls temporal patterning and is a probable microRNA target. Dev Cell 2003; 4:639-650.

93 Abrahante JE, Daul AL, Li M, et al. The Caenorhabditis elegans hunchback-like gene lin-57/hbl-1 controls developmental time and is regulated by microRNAs. Dev Cell 2003; 4:625-37.

94 Johnston RJ, Hobert O. A microRNA controlling left/right neuronal asymmetry in Caenorhabditis elegans. Nature 2003; 426: 845-9.

95 Park W, Li J, Song R, Messing J, Chen X. CARPEL FACTORY, a Dicer homolog, and HEN1, a novel protein, act in microRNA metabolism in Arabidopsis thaliana. Curr Biol 2002; 12:148495.
96 Chen X. A microRNA as a translational repressor of APETALA2 in Arabidopsis flower development. Science 2004; 303:2022-5.

97 Aukerman MJ, Sakai H. Regulation of flowering time and floral organ identity by a MicroRNA and its APETALA2-like target genes. Plant Cell 2003; 15:2730-41.

98 Palatnik JF, Allen E, Wu X, et al. Control of leaf morphogenesis by microRNAs. Nature 2003; 425:257-63.

99 Lai EC. Micro RNAs are complementary to 3' UTR sequence motifs that mediate negative post-transcriptional regulation. Nat Genet 2002; 30:363-4.

100 Stark A, Brennecke J, Russell RB, Cohen SM. Identification of Drosophila MicroRNA targets. PLoS Biol 2003; 1:E60.

101 Chang S, Johnston RJ Jr, Frokjaer-Jensen C, Lockery S, Hobert O. MicroRNAs act sequentially and asymmetrically to control chemosensory laterality in the nematode. Nature $2004 ; \mathbf{4 3 0}$ : 7859.

102 Xu P, Vernooy SY, Guo M, Hay BA. The Drosophila microRNA Mir-14 suppresses cell death and is required for normal fat metabolism. Curr Biol 2003; 13:790-5.

103 Brennecke J, Hipfner DR, Stark A, Russell RB, Cohen SM. Bantam encodes a developmentally regulated microRNA that controls cell proliferation and regulates the proapoptotic gene hid in Drosophila. Cell 2003; 113:25-36.

104Lewis BP, Shih IH, Jones-Rhoades MW, Bartel DP, Burge CB. Prediction of mammalian microRNA targets. Cell 2003; 115:78798.

105 Poy MN, Eliasson L, Krutzfeldt J, et al. A pancreatic islet-specific microRNA regulates insulin secretion. Nature 2004; 432: 226-230. 